\title{
Characteristics and Prognosis of Abdominal or Thoracic Aortic Aneurysm Patients Admitted to Intensive Care Units After Surgical Treatment: A Multicenter Retrospective Observational Study
}

This article was published in the following Dove Press journal:

International Journal of General Medicine

\author{
Qinchang Chen ${ }^{1,2, *}$ \\ Qingui Chen $\mathbb{1}^{3, *}$ \\ Yanchen $\mathrm{Ye}^{1,2}$ \\ Ridong $\mathrm{Wu}^{1,2}$ \\ Shenming Wang ${ }^{1,2}$ \\ Chen Yao ${ }^{1,2}$ \\ 'Department of Vascular Surgery, The \\ First Affiliated Hospital, Sun Yat-sen \\ University, Guangzhou, 510080, People's \\ Republic of China; ${ }^{2}$ National-Guangdong \\ Joint Engineering Laboratory for \\ Diagnosis and Treatment of Vascular \\ Diseases, The First Affiliated Hospital, \\ Sun Yat-sen University, Guangzhou, \\ 510080, People's Republic of China; \\ ${ }^{3}$ Department of Medical Intensive Care \\ Unit, The First Affiliated Hospital, Sun \\ Yat-sen University, Guangzhou, 510080, \\ People's Republic of China
}

*These authors contributed equally to this work
Correspondence: Shenming Wang; Chen Yao

Department of Vascular Surgery; National-Guangdong Joint Engineering Laboratory for Diagnosis and Treatment of Vascular Diseases, The First Affiliated Hospital, Sun Yat-sen University, No. 58 Zhongshan Road 2, Guangzhou, 510080, People's Republic of China Email shenmingwang@hotmail.com; yaochen@mail.sysu.edu.cn
Objective: To investigate the characteristics and prognosis of abdominal or thoracic aortic aneurysm (AAA or TAA) patients admitted to intensive care unit (ICU) postoperatively.

Methods: Patients admitted to ICU postoperatively with a primary diagnosis of AAA or TAA were screened in the eICU Collaborative Research Database, which contained data from multiple ICUs throughout the continental United States in 2014 and 2015. Baseline characteristics and comorbidities and were investigated and factors associated with ICU mortality were explored using univariable logistic regression. Receiver operating characteristic (ROC) curve analysis was performed to evaluate the prognosis predictive performance of the widely used severity scoring system APACHE IVa.

Results: A total of 974 patients including 677 AAA and 297 TAA patients admitted to ICU postoperatively were included. Compared with TAA, AAA patients had a significantly higher median age ( 72 versus 64 years, $\mathrm{P}<0.001$ ). 89.07\% AAA and $84.51 \%$ TAA patients underwent elective surgery $(\mathrm{P}=0.046), 8.71 \%$ AAA and $31.99 \%$ TAA patients were with aortic dissection $(\mathrm{P}<0.001)$, and $10.19 \%$ AAA and $2.36 \%$ TAA patients suffered from rupture of aortic aneurysm $(\mathrm{P}<0.001)$. Hypertension requiring treatment was the most common comorbidity $(57.31 \%$ for AAA and $61.95 \%$ for TAA). TAA patients had significantly higher ICU mortality $(9.43 \%$ versus $2.36 \%, \mathrm{P}<0.001)$ than AAA. Several factors were found to be significantly associated with ICU mortality, including urgent surgery, with aortic dissection, rupture of aortic aneurysm, TAA, and a higher APACHE IVa score on ICU admission. APACHE IVa showed a good predictive performance for ICU mortality with an area under the ROC curve of 0.9176 (95\% CI 0.8789-0.9390).

Conclusion: The prognosis of aortic aneurysm patients admitted to ICU postoperatively is yet to improve, and factors associated with prognosis are mainly related to the condition itself. APACHE IVa can be used for prognosis prediction.

Keywords: vascular surgery, intensive critical care, epidemiology

\section{Introduction}

Aortic aneurysm (AA) is the enlargement of the aorta defined as a segmental, fullthickness dilation of the blood vessel having at least a $50 \%$ increase in diameter compared with the expected normal diameter. ${ }^{1-3}$ It usually located in the abdominal aorta, but can also be located in the thoracic aorta. AA usually causes no symptoms; however, it is with an increased risk of aortic rupture due to weakness in the wall of the aorta and could be fatal when ruptured. ${ }^{4}$ 
As a clinically silent disease, the prevalence and incidence of abdominal aortic aneurysm (AAA) and thoracic aortic aneurysm (TAA) are low though varied between studies. AAA was reported to have a prevalence of $4-8 \%^{5-8}$ and TAA was $0.16-0.34 \% \%^{9,10}$ in screening studies. The relatively low prevalence and incidence make it difficult to conduct researches with enough sample sizes to obtain evidence, while obviously it is very important to have a working knowledge of the characteristics and prognosis of AA to aid with clinical decision-making.

In the past three decades, the greatest advance in the treatment of AA was the introduction of endovascular aneurysm repair (EVAR), which used stent grafts and caused a major paradigm shift in the field of AA surgery. ${ }^{11}$ For elective AAA repair, the mortality is $3-5 \%$ for open surgery but decreases to $0.5-2 \%$ for EVAR. ${ }^{12-14}$ However, the overall prognosis of AA is still not satisfactory, especially for ruptured AA. The 30-day and 90-day all-cause mortality rates were reported to be $22.9 \%$ and $33.3 \%$, respectively, for ruptured AAA patients after surgical treatment. ${ }^{15}$ To further improve the prognosis of AA, no doubt postoperative management should be improved. ${ }^{16}$

Although not all AA patients need intensive care unit (ICU) admissions after surgical treatment, ${ }^{17}$ subsequent ICU admissions postoperatively at least are not rare for patients with AAA or TAA. A study from Denmark reported that $62 \%$ of AAA patients after open AAA repair surgery had ICU stays $>24$ hours. ${ }^{18}$ A study investigated patients undergoing elective infrarenal EVAR between 2009 and 2015 and found that $57.3 \%$ were admitted to ICU after the surgery. ${ }^{19}$ In order to further improve the management of AA patients admitted to ICU postoperatively, an overview of their characteristics and risk profiles of prognosis is needed. However, as far as we know, a large-scale investigation on these patients is absent. Therefore, the study aimed to investigate the characteristics and prognosis of AAA or TAA patients admitted to ICU postoperatively.

\section{Methods}

\section{Data Source}

The study used data from the eICU Collaborative Research Database (version 2.0), which was made available largely by Philips Healthcare and collaborators at Massachusetts Institute of Technology (MIT) Laboratory for Computational Physiology. ${ }^{20}$ It consisted of data of patients admitted to one of 335 critical care units at 208 hospitals that participated in the Philips eICU program throughout the continental United States in 2014 and 2015. The database is released under the Health Insurance Portability and Accountability Act (HIPAA) safe harbor provision. The re-identification risk was certified as meeting safe harbor standards by Privacert (Cambridge, MA) (HIPAA Certification no. 1,031,219-2). Access to the database was approved after completing the CITI "Data or Specimens Only Research" course. This study was exempt from institutional review board approval due to the retrospective design, lack of direct patient intervention, and the security schema for the re-identification risk.

\section{Study Population}

Patients admitted to ICU postoperatively with a primary diagnosis of AAA or TAA were screened in the database. Data on primary diagnosis were extracted from the table "admissiondx" which contained the primary diagnosis for admission to the ICU per the Acute Physiology And Chronic Health Evaluation (APACHE) scoring criteria. AAA or TAA was identified based on structured text including "Aneurysm, abdominal aortic", "Aneurysm, abdominal aortic; with dissection", "Aneurysm, abdominal aortic; with rupture", "Aneurysm, thoracic aortic", "Aneurysm, thoracic aortic; with dissection", and "Aneurysm, thoracic aortic; with rupture". Detailed inclusion criteria were: 1) a primary diagnosis of AAA or TAA; 2) first ICU admission only (for patients who had more than 1 ICU admission during the same hospitalization); 3) admitted from operating room, recovery room, or post-anesthesia care unit (PACU); 4) age $\geq 18$ years. Detailed exclude criteria were: 1) instead of "admit", patients with an ICU stay type of "stepdown/other", "readmit for undo", "pre-admit", "transfer", or "readmit"; 2) patients who had more than 1 hospitalization records in the database; 3) length of ICU stay is missing. Flow chart of the study population is presented in Figure 1.

\section{Baseline Characteristics, Comorbidities, and Study Outcomes}

The following baseline characteristics were collected from the database, including age, sex, ethnicity, body mass index (BMI, defined as the body mass in kilograms divided by the square of the body height in meters), year of the hospital discharge date, type of surgery (elective or not), with or without aortic dissection, type of AA (ruptured or not), type 


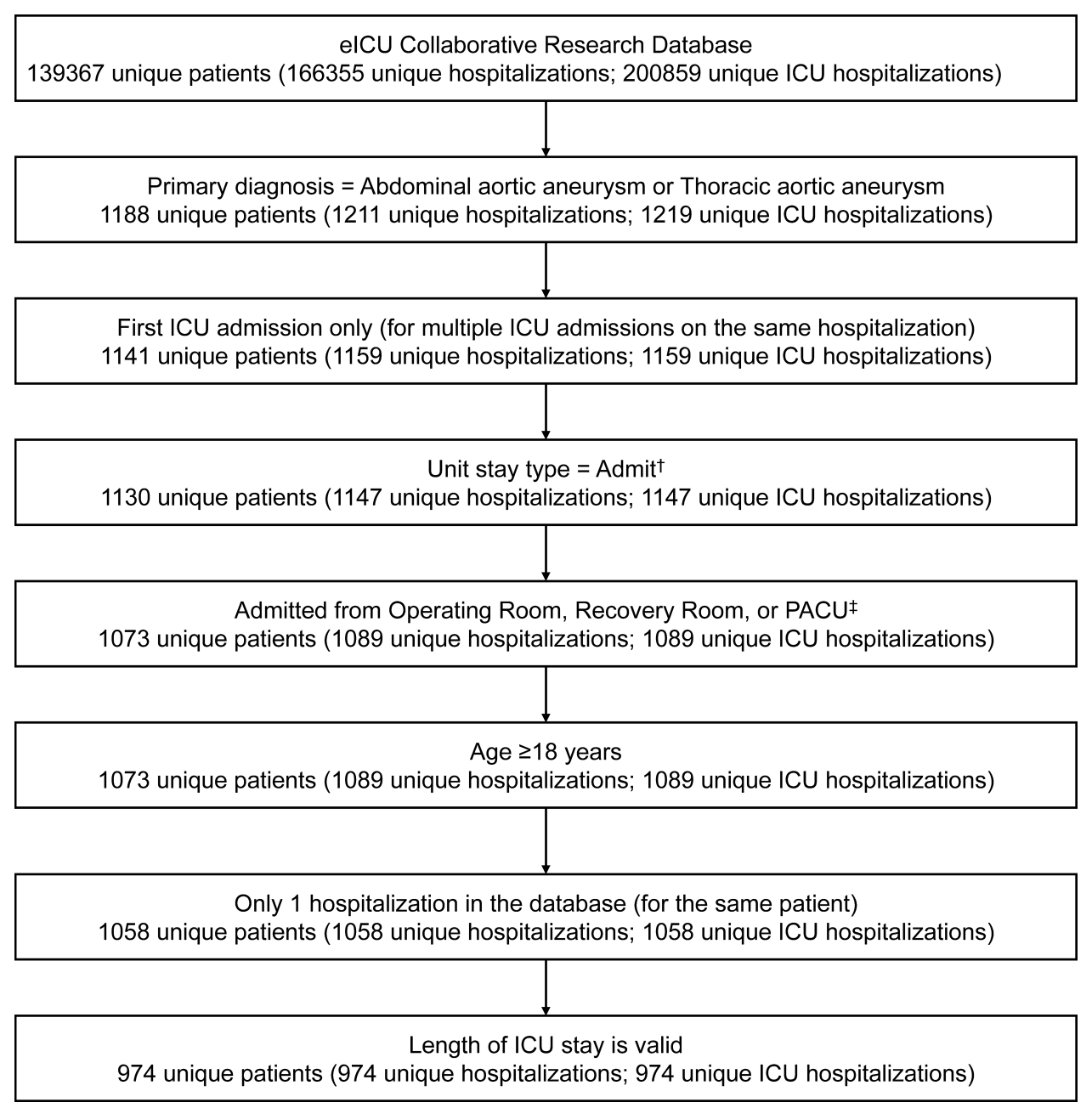

Figure I Flow chart of the study.

Notes: †Exclude unit stay type of readmit, stepdown/other, transfer. 拂clude admission from acute care/floor, direct admit, emergency department, floor, other hospital, other ICU, step-down unit (SDU).

Abbreviations: ICU, intensive care unit; PACU, post-anesthesia care unit.

of ICU, admitted location, acute physiology IVa score, APACHE IVa score, several lab parameters of the APACHE IVa scoring system, intubation, ventilation, and dialysis on the first day after ICU admission. Various comorbidities were also collected. ICU mortality was the primary outcome of the study. Length of ICU stay, hospital mortality, and length of hospital stay were also studied but only for description. All the variables above were extracted from the tables in the database, including "patient", "apachePatientResult", "apacheApsVar", and "pastHistory".

\section{Statistical Analysis}

Continuous variables were presented as median (25\% percentile $-75 \%$ percentile) and categorical variables were presented as number (percentage). Comparisons between groups were tested by Kruskal-Wallis $H$-test for continuous variables or Chi-squared test (or Fisher's exact test) for categorical variables. The study population was also categorized based on length of ICU stay ( $<2$ days, $2-7$ days, and $\geq 7$ days) and study outcomes of each category were described. Factors associated with ICU mortality were explored using univariable logistic regression. Kaplan-Meier curves for ICU mortality together with Log rank test were employed to compare the survival distribution of AAA and TAA. Receiver operating characteristic (ROC) curve analysis was performed to evaluate the prognosis predictive performance of APACHE IVa. A P value less than 0.05 was considered to indicate statistical significance. Empower(R) (www.empow erstats.com; X\&Y solutions, Inc., Boston, MA, USA) and $\mathrm{R}$ software, version 3.4.3 (http://www.r-project.org; R Foundation for Statistical Computing, Vienna, Austria) were used for statistical analyses. 


\section{Results}

\section{Baseline Characteristics and}

\section{Comorbidities of the Study Population}

A total of 974 AA patients admitted to ICU postoperatively were included finally. Among them, 549 (56.37\%) were AAA, 59 (6.06\%) were AAA with dissection, and 69 (7.08\%) were AAA with rupture; 195 (20.02\%) were TAA, $95(9.75 \%)$ were TAA with dissection, and $7(0.72 \%)$ were TAA with rupture. As presented in Table 1, the median age of the study population was 70 (62-77) years and 74.54\% (726/974) were male. Compared with TAA, AAA patients had a significantly higher median age ( 72 versus 64 years, $\mathrm{P}<0.001)$, and more patients were male $(79.17 \%$ versus $63.97 \%, \mathrm{P}<0.001)$. Proportions of patients who received elective surgery were broadly the same $(89.07 \%$ for AAA versus $84.51 \%$ for TAA, $\mathrm{P}=0.046) .8 .71 \%$ (59/677) AAA patients were with aortic dissection, while 31.99\% (95/ 297) for TAA patients. 10.19\% (69/677) AAA patients suffered from rupture of aortic aneurysm, but only $2.36 \%$ (7/297) for TAA patients. TAA patients had significantly higher acute physiology IVa score (41 versus $30, \mathrm{P}<0.001$ ) and APACHE IVa score (52 versus 45, $\mathrm{P}<0.001)$ on admission compared with AAA patients, and more needed intubation ( $45.45 \%$ versus $17.73 \%, \mathrm{P}<0.001$ ) or ventilation (44.11\% versus $20.38 \%, \mathrm{P}<0.001$ ). The above characteristics after stratified by (with or without) aortic dissection were presented in Table S1.

Comorbidities of the study population were presented in Table 2. The 5 most frequent comorbidities for AAA were hypertension requiring treatment (57.31\%), diabetes (16.10\%), chronic obstructive pulmonary disease (COPD, $14.62 \%)$, cancer $(13.00 \%)$, and myocardial infarction $(11.82 \%)$, while for TAA, the 5 most frequent comorbidities were hypertension requiring treatment $(61.95 \%)$, heart valve disease (14.81\%), renal insufficiency $(11.11 \%)$, diabetes $(10.44 \%)$, and congestive heart failure $(10.10 \%)$.

\section{Prognosis of the Study Population}

The overall ICU mortality for the study population was $4.52 \%$ (44/974), and the hospital mortality was 6.26\% (61/ 974). Compared with AAA, TAA patients had significantly higher ICU mortality $(9.43 \%$ versus $2.36 \%, \mathrm{P}<0.001)$ and hospital mortality $(12.12 \%$ versus $3.69 \%, \mathrm{P}<0.001)$. Consistent result was observed in Kaplan-Meier survival curves for ICU mortality (Figure 2), which showed a poorer survival in TAA patients compared with AAA patients ( $\log$ rank test, $\mathrm{P}=0.0072$ ). When the study population was categorized based on length of ICU stay $(<2$ days, 2-7 days, and $\geq 7$ days), patients with longer length of ICU stay had worse prognosis (Table 3 ). Prognosis of the study population after stratified by (with or without) aortic dissection was presented in Table S2, which showed higher ICU mortality in patients with aortic dissection compared to those without aortic dissection.

\section{Factors Associated with ICU Mortality}

Several baseline characteristics were found to be significantly associated with ICU mortality in univariable logistic regression (Table 4). Elective surgery was associated with reduced risk of ICU mortality (Odds ratio (OR) 0.22 , $95 \%$ confidence interval (CI) $0.12-0.42$ ), while with aortic dissection (OR 4.04, 95\% CI 2.16-7.57) and rupture of AA (OR 3.31, 95\% CI 1.53-7.18) were both associated with increased risk of ICU mortality. Compared with AAA, TAA was associated with an increased risk of ICU mortality (OR 4.30, 95\% CI 2.29-8.08). Acute physiology score IVa (OR 1.05 per 1 score increase, 95\% CI 1.04-1.06) and APACHE IVa score on admission (OR 1.05 per 1 score increase, 95\% CI 1.04-1.06) were also associated with poor prognosis. However, no comorbidities were found to be significantly associated with ICU mortality (Table 5).

\section{Prognosis Predictive Performance of APACHE IVa}

As presented in Table 6, APACHE IVa on ICU admission showed a good predictive performance for ICU mortality with an area under the ROC curve (AUC) of 0.9176 (95\% CI 0.8789-0.9390). Compared with AA patients without rupture, the predictive performance of APACHE IVa decreased in patients with rupture of AA (AUC 0.8795 versus 0.9158 ). TAA patients also saw a poorer predictive performance of APACHE IVa compared with AAA patients (AUC 0.8660 versus 0.9233).

\section{Discussion}

The study focused on AA patients who were admitted to ICU after surgical treatment and investigated the characteristics and prognosis of these patients. Predictors associated with prognosis and prognosis predictive performance of the widely used severity scoring system APACHE IVa were also explored. The main findings of the study were: 1) there were some great differences in 
Table I Baseline Characteristics of the Study Population

\begin{tabular}{|c|c|c|c|c|}
\hline Variables & All Patients ( $n=974)$ & $\begin{array}{l}\text { Abdominal Aortic Aneurysm } \\
(n=677)\end{array}$ & $\begin{array}{l}\text { Thoracic Aortic Aneurysm } \\
(n=297)\end{array}$ & $P$ value \\
\hline Age (years) & $70(62-77)$ & $72(65-78)$ & $64(54-74)$ & $<0.001$ \\
\hline Male & 726 (74.54\%) & $536(79.17 \%)$ & $190(63.97 \%)$ & $<0.001$ \\
\hline Ethnicity & & & & $<0.001$ \\
\hline Caucasian & $80 \mathrm{I}(82.24 \%)$ & 577 (85.23\%) & $224(75.42 \%)$ & \\
\hline African American & $96(9.86 \%)$ & 47 (6.94\%) & $49(16.50 \%)$ & \\
\hline Asian & $12(1.23 \%)$ & 7 (1.03\%) & $5(1.68 \%)$ & \\
\hline Hispanic & $12(1.23 \%)$ & $10(1.48 \%)$ & $2(0.67 \%)$ & \\
\hline Native American & $3(0.31 \%)$ & $0(0.00 \%)$ & $3(1.01 \%)$ & \\
\hline Other/Unknown & $50(5.13 \%)$ & $36(5.32 \%)$ & 14 (4.7I\%) & \\
\hline BMI $\left(\mathrm{kg} / \mathrm{m}^{2}\right)$ & $27.59(24.22-31.46)$ & $27.46(24.20-31.10)$ & $28.04(24.28-32.92)$ & 0.129 \\
\hline $\begin{array}{l}\text { Year of the hospital discharge } \\
\text { date }\end{array}$ & & & & 0.887 \\
\hline 2014 & 482 (49.49\%) & 334 (49.34\%) & I 48 (49.83\%) & \\
\hline 2015 & 492 (50.5 I\%) & $343(50.66 \%)$ & 149 (50.17\%) & \\
\hline Elective surgery & $854(87.68 \%)$ & 603 (89.07\%) & $25 I(84.51 \%)$ & 0.046 \\
\hline With aortic dissection & 154 (I5.8I\%) & 59 (8.7I\%) & 95 (31.99\%) & $<0.001$ \\
\hline Rupture of aortic aneurysm & $76(7.80 \%)$ & $69(10.19 \%)$ & 7 (2.36\%) & $<0.001$ \\
\hline Type of ICU & & & & $<0.001$ \\
\hline Med-Surg ICU & $287(29.47 \%)$ & $232(34.27 \%)$ & 55 (18.52\%) & \\
\hline CSICU & I54 (I5.8I\%) & II 15 (I6.99\%) & $39(13.13 \%)$ & \\
\hline CTICU & I84 (I8.89\%) & 87 (12.85\%) & 97 (32.66\%) & \\
\hline CCU-CTICU & $169(17.35 \%)$ & 95 (14.03\%) & 74 (24.92\%) & \\
\hline SICU & $124(12.73 \%)$ & 97 (14.33\%) & 27 (9.09\%) & \\
\hline Cardiac ICU & $30(3.08 \%)$ & 27 (3.99\%) & $3(1.01 \%)$ & \\
\hline Neuro ICU & $20(2.05 \%)$ & $18(2.66 \%)$ & $2(0.67 \%)$ & \\
\hline MICU & $6(0.62 \%)$ & $6(0.89 \%)$ & $0(0.00 \%)$ & \\
\hline Location admitted from & & & & 0.079 \\
\hline Operating Room & 767 (78.75\%) & $523(77.25 \%)$ & $244(82.15 \%)$ & \\
\hline Recovery Room & $169(17.35 \%)$ & 122 (I8.02\%) & 47 (15.82\%) & \\
\hline PACU & $38(3.90 \%)$ & $32(4.73 \%)$ & $6(2.02 \%)$ & \\
\hline Acute physiology IVa score & $32(24-47.75)$ & $30(22-42)$ & $4 \mid(29-6 I)$ & $<0.001$ \\
\hline APACHE IVa score & $47(37-62)$ & $45(36-57)$ & $52(37.25-75)$ & $<0.001$ \\
\hline \multicolumn{5}{|l|}{$\begin{array}{l}\text { Lab variables in APACHE IVa } \\
\text { score }\end{array}$} \\
\hline Temperature $\left({ }^{\circ} \mathrm{C}\right)$ & $36.3(35.8-36.6)$ & $36.3(36.0-36.6)$ & $36.1(35.5-36.6)$ & $<0.001$ \\
\hline Mean blood pressure $(\mathrm{mmHg})$ & $68(56-132)$ & $71(57-133)$ & $62(52-128)$ & $<0.001$ \\
\hline Heart rate $(/ \mathrm{min})$ & $96(59-109)$ & $96(58-108)$ & $99(82-112)$ & 0.006 \\
\hline Respiratory rate (/min) & $13(9-3 \mid)$ & $14(9-32)$ & $12(8-30)$ & 0.004 \\
\hline $\mathrm{FiO}_{2}(\%)$ & $50(40-80)$ & $50(40-80)$ & $60(40-87.50)$ & 0.087 \\
\hline $\mathrm{PaO}_{2}(\mathrm{mmHg})$ & $102.90(80.00-154.45)$ & $103.00(80.00-159.00)$ & $101.65(79.00-151.00)$ & 0.553 \\
\hline $\mathrm{PaCO}_{2}(\mathrm{mmHg})$ & $42.00(37.00-46.25)$ & $42.00(37.00-46.10)$ & $42.00(37.38-46.77)$ & 0.875 \\
\hline Arterial pH & $7.36(7.31-7.40)$ & $7.34(7.30-7.39)$ & $7.37(7.33-7.4 I)$ & 0.002 \\
\hline Sodium (mEq/L) & $139(|36-14|)$ & $139(|36-14|)$ & $140(138-143.5)$ & $<0.001$ \\
\hline Urine output $(\mathrm{mL} / 24 \mathrm{~h})$ & $1621.99(987.60-2586.69)$ & I585.0| (907.72-2472.42) & $1691.02(1172.02-2791.11)$ & 0.096 \\
\hline Creatinine (mg/dL) & $1.00(0.80-1.40)$ & $\mathrm{I} .00(0.80-1.36)$ & $1.02(0.75-1.50)$ & 0.888 \\
\hline Blood urea nitrogen $(\mathrm{mg} / \mathrm{dL})$ & $17(13-23)$ & $16.5(13-22)$ & $17(13-24.5)$ & 0.171 \\
\hline
\end{tabular}


Table I (Continued).

\begin{tabular}{|c|c|c|c|c|}
\hline Variables & All Patients $(n=974)$ & $\begin{array}{l}\text { Abdominal Aortic Aneurysm } \\
(n=677)\end{array}$ & $\begin{array}{l}\text { Thoracic Aortic Aneurysm } \\
(n=297)\end{array}$ & $P$ value \\
\hline Glucose (mg/dL) & $14 \mid(105-182)$ & $138(107.25-180)$ & I5I (99-184.5) & 0.564 \\
\hline Albumin $(g / d L)$ & $2.90(2.50-3.30)$ & $2.90(2.50-3.30)$ & $2.90(2.48-3.30)$ & 0.930 \\
\hline Bilirubin (mg/dL) & $0.90(0.50-1.55)$ & $0.80(0.50-1.33)$ & $1.10(0.60-1.90)$ & 0.008 \\
\hline Hematocrit (\%) & $31.50(27.40-35.60)$ & $32.55(28.83-36.50)$ & $29.10(25.50-33.20)$ & $<0.001$ \\
\hline WBC $\left(\times 1000 / \mathrm{mm}^{3}\right)$ & $10.50(7.98-14.70)$ & $10.30(7.90-14.10)$ & $12.20(8.20-16.60)$ & 0.003 \\
\hline Intubated & $255(26.18 \%)$ & $120(17.73 \%)$ & 135 (45.45\%) & $<0.001$ \\
\hline Ventilation & $269(27.62 \%)$ & I 38 (20.38\%) & $|3|(44 .|| \%)$ & $<0.001$ \\
\hline Dialysis & $7(0.72 \%)$ & $4(0.59 \%)$ & $3(1.01 \%)$ & 0.442 \\
\hline
\end{tabular}

Note: $P$ value $<0.05$ was presented in bold.

Abbreviations: BMI, body mass index; ICU, intensive care unit; Med-Surg ICU, medical-surgical intensive care unit; CSICU, cardiac surgery intensive care unit; CTICU, cardiothoracic intensive care unit; CCU-CTICU, coronary care unit-cardiothoracic intensive care unit; SICU, surgical intensive care unit; MICU, medical intensive care unit; PACU, post-anesthesia care unit; APACHE, Acute Physiology And Chronic Health Evaluation; $\mathrm{FiO}_{2}$, fraction of inspired oxygen; $\mathrm{PaO}_{2}$, partial pressure of oxygen; $\mathrm{PaCO}_{2}$, partial pressure of carbon dioxide; WBC, white blood cell.

Table 2 Comorbidities of the Study Population

\begin{tabular}{|c|c|c|c|c|}
\hline Variables & $\begin{array}{l}\text { All Patients } \\
(n=974)\end{array}$ & $\begin{array}{l}\text { Abdominal Aortic Aneurysm } \\
(n=677)\end{array}$ & $\begin{array}{l}\text { Thoracic Aortic Aneurysm } \\
(n=297)\end{array}$ & $P$ value \\
\hline $\begin{array}{l}\text { History of coronary artery bypass } \\
\text { surgery }\end{array}$ & 87 (8.93\%) & $72(10.64 \%)$ & $15(5.05 \%)$ & 0.005 \\
\hline History of angina & $24(2.46 \%)$ & $20(2.95 \%)$ & $4(1.35 \%)$ & 0.179 \\
\hline History of myocardial infarction & $96(9.86 \%)$ & $80(11.82 \%)$ & 16 (5.39\%) & 0.002 \\
\hline $\begin{array}{l}\text { History of percutaneous coronary } \\
\text { intervention }\end{array}$ & $63(6.47 \%)$ & $51(7.53 \%)$ & $12(4.04 \%)$ & 0.041 \\
\hline History of congestive heart failure & $66(6.78 \%)$ & $36(5.32 \%)$ & $30(10.10 \%)$ & 0.006 \\
\hline History of atrial fibrillation & $80(8.21 \%)$ & $56(8.27 \%)$ & $24(8.08 \%)$ & 0.920 \\
\hline History of AICD/pacemaker & $25(2.57 \%)$ & $16(2.36 \%)$ & 9 (3.03\%) & 0.518 \\
\hline $\begin{array}{l}\text { History of hypertension requiring } \\
\text { treatment }\end{array}$ & $572(58.73 \%)$ & $388(57.31 \%)$ & $184(61.95 \%)$ & 0.176 \\
\hline History of heart valve disease & $7 \mathrm{I}(7.29 \%)$ & 27 (3.99\%) & 44 (I4.8I\%) & $<0.001$ \\
\hline History of COPD & 125 (I2.83\%) & 99 (14.62\%) & $26(8.75 \%)$ & 0.012 \\
\hline History of asthma & 35 (3.59\%) & $20(2.95 \%)$ & $15(5.05 \%)$ & 0.106 \\
\hline History of respiratory failure & $5(0.5 \mid \%)$ & $4(0.59 \%)$ & I (0.34\%) & 1.000 \\
\hline $\begin{array}{l}\text { History of restrictive pulmonary } \\
\text { disease }\end{array}$ & $7(0.72 \%)$ & $4(0.59 \%)$ & $3(1.01 \%)$ & 0.442 \\
\hline History of cirrhosis & $16(1.64 \%)$ & 13 (1.92\%) & $3(1.01 \%)$ & 0.416 \\
\hline History of peptic ulcer disease & $22(2.26 \%)$ & $17(2.51 \%)$ & $5(1.68 \%)$ & 0.492 \\
\hline History of renal insufficiency & $86(8.83 \%)$ & $53(7.83 \%)$ & $33(11.11 \%)$ & 0.096 \\
\hline History of dialysis & $8(0.82 \%)$ & $5(0.74 \%)$ & $3(1.01 \%)$ & 0.705 \\
\hline $\begin{array}{l}\text { History of non-cancerous hematology } \\
\text { disease }\end{array}$ & $5(0.5 \mid \%)$ & $4(0.59 \%)$ & I (0.34\%) & 1.000 \\
\hline History of anemia & $3(0.31 \%)$ & $2(0.30 \%)$ & I (0.34\%) & 1.000 \\
\hline History of ITP & I (0.10\%) & I (0.15\%) & $0(0.00 \%)$ & 1.000 \\
\hline History of peripheral vascular disease & $88(9.03 \%)$ & $72(10.64 \%)$ & $16(5.39 \%)$ & 0.009 \\
\hline History of VTE & $40(4.11 \%)$ & $24(3.55 \%)$ & $16(5.39 \%)$ & 0.182 \\
\hline History of DVT & $26(2.67 \%)$ & $18(2.66 \%)$ & $8(2.69 \%)$ & 1.000 \\
\hline History of PE & $18(1.85 \%)$ & $10(1.48 \%)$ & 8 (2.69\%) & 0.203 \\
\hline History of diabetes & 140 (I4.37\%) & $109(16.10 \%)$ & 31 (10.44\%) & 0.020 \\
\hline History of hyperthyroidism & $6(0.62 \%)$ & $4(0.59 \%)$ & $2(0.67 \%)$ & 1.000 \\
\hline
\end{tabular}

(Continued) 
Table 2 (Continued).

\begin{tabular}{|c|c|c|c|c|}
\hline Variables & $\begin{array}{l}\text { All Patients } \\
(n=974)\end{array}$ & $\begin{array}{l}\text { Abdominal Aortic Aneurysm } \\
(n=677)\end{array}$ & $\begin{array}{l}\text { Thoracic Aortic Aneurysm } \\
(n=297)\end{array}$ & $\mathbf{P}$ value \\
\hline History of hypothyroidism & 68 (6.98\%) & 43 (6.35\%) & 25 (8.42\%) & 0.244 \\
\hline History of stroke & 57 (5.85\%) & 37 (5.47\%) & $20(6.73 \%)$ & 0.437 \\
\hline History of TIA & 34 (3.49\%) & $23(3.40 \%)$ & II (3.70\%) & 0.810 \\
\hline History of dementia & $3(0.31 \%)$ & $3(0.44 \%)$ & $0(0.00 \%)$ & 0.557 \\
\hline History of neuromuscular disease & I $(0.10 \%)$ & I (0.15\%) & $0(0.00 \%)$ & 1.000 \\
\hline History of seizures & $19(1.95 \%)$ & $12(1.77 \%)$ & $7(2.36 \%)$ & 0.616 \\
\hline AIDS & $4(0.41 \%)$ & $3(0.44 \%)$ & I (0.34\%) & 1.000 \\
\hline Immunosuppression within past 6 months & $4(0.41 \%)$ & $3(0.44 \%)$ & I (0.34\%) & 1.000 \\
\hline History of rheumatic disease & $13(1.33 \%)$ & II (I.62\%) & $2(0.67 \%)$ & 0.364 \\
\hline History of rheumatoid arthritis & $13(1.33 \%)$ & II (I.62\%) & $2(0.67 \%)$ & 0.364 \\
\hline History of SLE & I $(0.10 \%)$ & I (0.15\%) & $0(0.00 \%)$ & 1.000 \\
\hline History of heart transplant & $2(0.21 \%)$ & $2(0.30 \%)$ & $0(0.00 \%)$ & 1.000 \\
\hline History of liver transplant & $\mathrm{I}(0.10 \%)$ & I (0.15\%) & $0(0.00 \%)$ & 1.000 \\
\hline History of renal transplant & $4(0.41 \%)$ & $2(0.30 \%)$ & $2(0.67 \%)$ & 0.590 \\
\hline History of cancer & 116 (11.91\%) & $88(13.00 \%)$ & $28(9.43 \%)$ & 0.113 \\
\hline History of metastases cancer & $4(0.41 \%)$ & $2(0.30 \%)$ & $2(0.67 \%)$ & 0.590 \\
\hline History of head neck cancer & $6(0.62 \%)$ & $6(0.89 \%)$ & $0(0.00 \%)$ & 0.186 \\
\hline History of esophagus cancer & $4(0.41 \%)$ & $4(0.59 \%)$ & $0(0.00 \%)$ & 0.320 \\
\hline History of lung cancer & $16(1.64 \%)$ & $14(2.07 \%)$ & $2(0.67 \%)$ & 0.170 \\
\hline History of breast cancer & $12(1.23 \%)$ & $6(0.89 \%)$ & $6(2.02 \%)$ & 0.203 \\
\hline History of liver cancer & $2(0.21 \%)$ & I (0.15\%) & I (0.34\%) & 0.517 \\
\hline History of stomach cancer & $3(0.31 \%)$ & $2(0.30 \%)$ & I (0.34\%) & 1.000 \\
\hline History of bladder cancer & $10(1.03 \%)$ & $9(1.33 \%)$ & I (0.34\%) & 0.298 \\
\hline History of kidney cancer & $10(1.03 \%)$ & 5 (0.74\%) & $5(1.68 \%)$ & 0.183 \\
\hline History of colon cancer & $5(0.51 \%)$ & $5(0.74 \%)$ & $0(0.00 \%)$ & 0.331 \\
\hline History of melanoma & $8(0.82 \%)$ & $6(0.89 \%)$ & $2(0.67 \%)$ & 1.000 \\
\hline History of ovary cancer & $2(0.21 \%)$ & I (0.15\%) & I (0.34\%) & 0.517 \\
\hline History of uterus cancer & I (0.10\%) & $\mathrm{I}(0.15 \%)$ & $0(0.00 \%)$ & 1.000 \\
\hline History of prostate cancer & $22(2.26 \%)$ & $17(2.51 \%)$ & $5(1.68 \%)$ & 0.492 \\
\hline History of chemotherapy & $3(0.3 \mid \%)$ & $2(0.30 \%)$ & I (0.34\%) & 1.000 \\
\hline Radiation therapy within past 6 months & $2(0.21 \%)$ & $2(0.30 \%)$ & $0(0.00 \%)$ & 1.000 \\
\hline
\end{tabular}

Note: $P$ value $<0.05$ was presented in bold.

Abbreviations: AICD, automatic implantable cardioverter defibrillator; COPD, chronic obstructive pulmonary disease; ITP, immune thrombocytopenic purpura; VTE, venous thromboembolism; DVT, deep vein thrombosis; PE, pulmonary embolism; TIA, transient ischemic attack; AIDS, acquired immune deficiency syndrome; SLE, systemic lupus erythematosus.

baseline characteristics and comorbidities between AAA and TAA patients who were admitted to ICU postoperatively; 2) prognosis of these patients is yet to improve, especially for TAA patients; 3) instead of comorbidities, factors associated with prognosis were mainly related to the condition itself; 4) APACHE IVa showed good prognosis predictive performance. Based on a relatively large sample size and the recent data (year 2014 and 2015), these findings provided an updated overview of AA patients (either AAA or TAA) admitted to ICU after receiving surgical treatment, which might help to improve postoperative management of AA patients and therefore to further improve their prognosis.

Compared with most other studies, the baseline characteristics and comorbidities of AA patients in our study were broadly similar, including an advanced age, predominantly males, and a higher prevalence of hypertension comorbidity. For example, in a cohort from Japan who underwent open or endovascular repair for $\mathrm{AAA},{ }^{21}$ only $1.4 \%$ (14/999) of patients aged 50 years or younger, $85.3 \%$ (852/999) were male, and 79.9\% (798/999) had hypertension. In a retrospective cross-sectional study from Iran, ${ }^{22}$ 


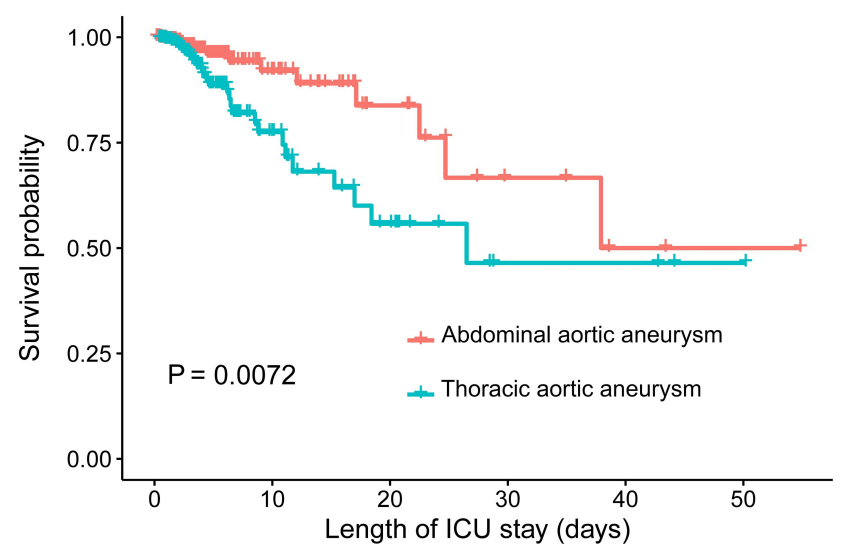

\begin{tabular}{|lccccc} 
Number at risk & & & \\
677 & 37 & 13 & 5 & 2 & 1 \\
297 & 30 & 12 & 3 & 3 & 1 \\
\hline 0 & 10 & $\begin{array}{c}20 \\
\text { Length of ICU stay (days) }\end{array}$ & 50
\end{tabular}

Figure 2 Kaplan-Meier survival curves for ICU mortality. Abbreviation: ICU, intensive care unit.

the mean age of AAA patients in emergency department was $68.11 \pm 11.98$ years, $84 \%$ were male, and $51.6 \%$ had hypertension. In a cohort of 107 untreated TAA patients, ${ }^{23}$ the average age was 59.3 years, $73.8 \%$ (79/107) patients were male, and $47 \%$ had diastolic hypertension. Similarities between these studies might be related to the nature of the diseases, but it should be noticed that in our study, the study population were only AA patients who survived after the surgical treatment and needed an admission to ICU. This might explain the slight difference in these characteristics between studies. A recent study found that female AAA patients might have a higher risk of rupture due to gender differences in morphological and hemodynamic characteristics of $\mathrm{AAA},{ }^{24}$ and considering that patients with rupture were more likely to be admitted to ICU after surgery, ${ }^{17}$ our study thus included more female AAA patients. However, given the limited sample size of all these studies, variations in statistics of these characteristics could also explain the difference. In terms of ethnicity, most patients were Caucasian in our study, which was consistent with several other studies ${ }^{25-27}$ that supported Caucasian race might be a risk factor for the development of AAA. Researches that compared AAA with TAA were rare, making it impossible to compare our results with others. In our study, the TAA patients were younger, with a much lower proportion of rupture, and different patterns of comorbidities when compared with AAA patients. These differences might be related to the "selection bias" of our study population. As mentioned above, only patients who underwent a surgery, survived, and at the same time needed to be admitted to ICU would be included in our study. Considering TAA had worse prognosis, ${ }^{28}$ it is not strange to find that patients who survived and therefore were enrolled were younger and presented a different pattern of comorbidities compared with the AAA patients who were much elder.

Reports about the prognosis of AA patients varied. A study included 138 ruptured AAA patients who received open surgical repair and survived at least 48 hours in ICU

Table 3 Clinical Outcomes of the Study Population Stratified by Length of ICU Stay

\begin{tabular}{|c|c|c|c|c|}
\hline Clinical Outcomes & $\begin{array}{l}\text { All Patients } \\
(n=974)\end{array}$ & $\begin{array}{l}\text { Abdominal Aortic Aneurysm } \\
(n=677)\end{array}$ & $\begin{array}{l}\text { Thoracic Aortic Aneurysm } \\
(n=297)\end{array}$ & P value \\
\hline $\begin{array}{l}\text { ICU mortality } \\
\text { Length of ICU stay }<2 \text { days } \\
\text { Length of ICU stay } 2-7 \text { days } \\
\text { Length of ICU stay } \geq 7 \text { days } \\
P \text { value }\end{array}$ & $\begin{array}{l}44(4.52 \%) \\
9(1.56 \%) \\
20(6.58 \%) \\
15(15.96 \%) \\
<0.001\end{array}$ & $\begin{array}{l}16(2.36 \%) \\
5(1.08 \%) \\
5(3.07 \%) \\
6(12.00 \%) \\
<0.001\end{array}$ & $\begin{array}{l}28(9.43 \%) \\
4(3.57 \%) \\
15(10.64 \%) \\
9(20.45 \%) \\
0.004\end{array}$ & $<0.001$ \\
\hline $\begin{array}{l}\text { Hospital mortality } \\
\text { Length of ICU stay }<2 \text { days } \\
\text { Length of ICU stay } 2-7 \text { days } \\
\text { Length of ICU stay } \geq 7 \text { days } \\
\text { P value }\end{array}$ & $\begin{array}{l}61(6.26 \%) \\
12(2.08 \%) \\
29(9.54 \%) \\
20(21.28 \%) \\
<0.001\end{array}$ & $\begin{array}{l}25(3.69 \%) \\
7(1.51 \%) \\
10(6.13 \%) \\
8(16.00 \%) \\
<0.001\end{array}$ & $\begin{array}{l}36(12.12 \%) \\
5(4.46 \%) \\
19(13.48 \%) \\
12(27.27 \%) \\
<0.001\end{array}$ & $<0.001$ \\
\hline $\begin{array}{l}\text { Length of ICU stay (days) } \\
\text { Length of hospital stay (days) }\end{array}$ & $\begin{array}{l}1.54(0.99-3.24) \\
4.79(2.10-8.33)\end{array}$ & $\begin{array}{l}1.17(0.94-2.68) \\
3.25(1.40-7.33)\end{array}$ & $\begin{array}{l}2.37(1.36-4.67) \\
6.40(4.36-10.44)\end{array}$ & $\begin{array}{l}<0.001 \\
<0.001\end{array}$ \\
\hline
\end{tabular}

Note: $P$ value $<0.05$ was presented in bold.

Abbreviation: ICU, intensive care unit. 
Table 4 Univariable Logistic Regression Analysis of Baseline Characteristics Associated with ICU Mortality

\begin{tabular}{|c|c|c|c|}
\hline Variables & OR & $95 \% \mathrm{Cl}$ & $P$ value \\
\hline Age (years) & 0.98 & $0.95-1.00$ & 0.0356 \\
\hline \multicolumn{4}{|l|}{ Sex } \\
\hline Male & 1.0 & & \\
\hline Female & 1.55 & $0.82-2.94$ & 0.1818 \\
\hline \multicolumn{4}{|l|}{ Ethnicity } \\
\hline Caucasian & 1.0 & & \\
\hline African American & 1.28 & $0.49-3.36$ & 0.6177 \\
\hline Asian & 0.00 & $0.00-\operatorname{lnf}$ & 0.9845 \\
\hline Hispanic & 4.65 & $0.98-22.10$ & 0.0530 \\
\hline Native American & 46.55 & $4 .|2-526.4|$ & 0.0019 \\
\hline Other/Unknown & 0.97 & $0.23-4.16$ & 0.9670 \\
\hline BMI $\left(\mathrm{kg} / \mathrm{m}^{2}\right)$ & 0.99 & $0.95-1.04$ & 0.8133 \\
\hline \multicolumn{4}{|l|}{ Year of the hospital discharge date } \\
\hline 2014 & 1.0 & & \\
\hline 2015 & 1.08 & $0.59-1.97$ & 0.8112 \\
\hline \multicolumn{4}{|l|}{ Elective surgery } \\
\hline No & 1.0 & & \\
\hline Yes & 0.22 & $0.12-0.42$ & $<0.0001$ \\
\hline \multicolumn{4}{|l|}{ With aortic dissection } \\
\hline No & 1.0 & & \\
\hline Yes & 4.04 & $2.16-7.57$ & $<0.0001$ \\
\hline \multicolumn{4}{|l|}{ Rupture of aortic aneurysm } \\
\hline No & 1.0 & & \\
\hline Yes & 3.31 & $1.53-7.18$ & 0.0024 \\
\hline \multicolumn{4}{|l|}{ Type of ICU } \\
\hline Med-Surg ICU & 1.0 & & \\
\hline CSICU & 1.78 & $0.79-4.01$ & 0.1627 \\
\hline CTICU & 0.71 & $0.27-1.90$ & 0.4966 \\
\hline CCU-CTICU & 0.64 & $0.22-1.84$ & 0.4088 \\
\hline SICU & 1.26 & $0.49-3.24$ & 0.6302 \\
\hline Cardiac ICU & 0.00 & $0.00-\operatorname{lnf}$ & 0.9840 \\
\hline Neuro ICU & 1.11 & $0.14-8.94$ & 0.9224 \\
\hline MICU & 0.00 & $0.00-\operatorname{lnf}$ & 0.9928 \\
\hline \multicolumn{4}{|l|}{ Location admitted from for ICU } \\
\hline Operating Room & 1.0 & & \\
\hline Recovery Room & 0.57 & $0.22-1.47$ & 0.2430 \\
\hline PACU & 0.00 & $0.00-\operatorname{lnf}$ & 0.9818 \\
\hline \multicolumn{4}{|l|}{ Type of aortic aneurysm } \\
\hline Abdominal aortic aneurysm & 1.0 & & \\
\hline Thoracic aortic aneurysm & 4.30 & $2.29-8.08$ & $<0.0001$ \\
\hline \multicolumn{4}{|l|}{ Type of aortic aneurysm } \\
\hline Abdominal aortic aneurysm & 1.0 & & \\
\hline $\begin{array}{l}\text { Abdominal aortic aneurysm } \\
\text { with dissection }\end{array}$ & 4.15 & $1.04-16.49$ & 0.0434 \\
\hline
\end{tabular}

(Continued)
Table 4 (Continued).

\begin{tabular}{|c|c|c|c|}
\hline Variables & OR & $95 \% \mathrm{Cl}$ & $P$ value \\
\hline $\begin{array}{l}\text { Abdominal aortic aneurysm } \\
\text { with rupture }\end{array}$ & 7.37 & $2.40-22.63$ & 0.0005 \\
\hline Thoracic aortic aneurysm & 4.19 & $1.57-11.15$ & 0.0042 \\
\hline $\begin{array}{l}\text { Thoracic aortic aneurysm with } \\
\text { dissection }\end{array}$ & 14.52 & $5.74-36.70$ & $<0.0001$ \\
\hline $\begin{array}{l}\text { Thoracic aortic aneurysm with } \\
\text { rupture }\end{array}$ & 58.07 & $10.91-309.22$ & $<0.0001$ \\
\hline Acute physiology score IVa & 1.05 & $1.04-1.06$ & $<0.0001$ \\
\hline APACHE IVa score & 1.05 & $1.04-1.06$ & $<0.0001$ \\
\hline Lab variables in APACHE IVa score & & & \\
\hline Temperature $\left({ }^{\circ} \mathrm{C}\right)$ & 0.58 & $0.47-0.72$ & $<0.0001$ \\
\hline Mean blood pressure $(\mathrm{mmHg})$ & 1.00 & $0.99-1.00$ & 0.2350 \\
\hline Heart rate $(/ \mathrm{min})$ & 1.03 & $1.02-1.04$ & $<0.0001$ \\
\hline Respiratory rate (/min) & 0.97 & $0.95-1.00$ & 0.0356 \\
\hline $\mathrm{FiO}_{2}(\%)$ & 1.03 & $1.02-1.05$ & $<0.0001$ \\
\hline $\mathrm{PaO}_{2}(\mathrm{mmHg})$ & 1.00 & $0.99-1.00$ & 0.6106 \\
\hline $\mathrm{PaCO}_{2}(\mathrm{mmHg})$ & 1.01 & $0.97-1.06$ & 0.6192 \\
\hline Arterial $\mathrm{pH}$ & 0.00 & $0.00-0.00$ & $<0.0001$ \\
\hline Sodium (mEq/L) & 1.19 & $1.13-1.26$ & $<0.0001$ \\
\hline Urine output $(\mathrm{mL} / 24 \mathrm{~h})$ & 1.00 & $1.00-1.00$ & 0.0021 \\
\hline Creatinine $(\mathrm{mg} / \mathrm{dL})$ & 1.75 & $1.39-2.20$ & $<0.0001$ \\
\hline Blood urea nitrogen $(\mathrm{mg} / \mathrm{dL})$ & 1.06 & $1.03-1.08$ & $<0.0001$ \\
\hline Glucose (mg/dL) & 1.01 & $1.00-1.01$ & 0.0005 \\
\hline Albumin $(\mathrm{g} / \mathrm{dL})$ & 0.22 & $0.11-0.44$ & $<0.0001$ \\
\hline Bilirubin $(\mathrm{mg} / \mathrm{dL})$ & 1.83 & $1.30-2.57$ & 0.0005 \\
\hline Hematocrit (\%) & 0.82 & $0.77-0.87$ & $<0.0001$ \\
\hline WBC $\left(\times 1000 / \mathrm{mm}^{3}\right)$ & 1.05 & $1.00-1.09$ & 0.0284 \\
\hline \multicolumn{4}{|l|}{ Intubated } \\
\hline No & 1.0 & & \\
\hline Yes & 8.45 & $4.28-16.69$ & $<0.0001$ \\
\hline \multicolumn{4}{|l|}{ Ventilation } \\
\hline No & 1.0 & & \\
\hline Yes & 19.16 & $8.00-45.92$ & $<0.0001$ \\
\hline \multicolumn{4}{|l|}{ Dialysis } \\
\hline No & 1.0 & & \\
\hline Yes & 3.58 & $0.42-30.41$ & 0.2424 \\
\hline
\end{tabular}

Note: $P$ value $<0.05$ was presented in bold.

Abbreviations: ICU, intensive care unit; OR, odds ratio; $\mathrm{Cl}$, confidence interval; Inf, infinity; BMI, body mass index; Med-Surg ICU, medical-surgical intensive care unit; CSICU, cardiac surgery intensive care unit; CTICU, cardiothoracic intensive care unit; CCU-CTICU, coronary care unit-cardiothoracic intensive care unit; SICU, surgical intensive care unit; MICU, medical intensive care unit; PACU, post-anesthesia care unit; APACHE, Acute Physiology And Chronic Health Evaluation; $\mathrm{FiO}_{2}$, fraction of inspired oxygen; $\mathrm{PaO}_{2}$, partial pressure of oxygen; $\mathrm{PaCO}_{2}$, partial pressure of carbon dioxide; WBC, white blood cell.

between 1999 and 2003 reported a 30-day mortality of $22 \%{ }^{29}$ This mortality rate was much higher than that of our study population, which might be related to the different study population since ruptured AA had a significantly 
Table 5 Univariable Logistic Regression Analysis of Comorbidities Associated with ICU Mortality

\begin{tabular}{|c|c|c|c|}
\hline Variables & OR* & $95 \% \mathrm{Cl}$ & $P$ value \\
\hline $\begin{array}{l}\text { History of coronary artery bypass } \\
\text { surgery }\end{array}$ & 0.23 & $0.03-1.68$ & 0.1467 \\
\hline History of angina & 0.92 & $0.12-6.95$ & 0.9332 \\
\hline History of myocardial infarction & 0.20 & $0.03-1.50$ & 0.1186 \\
\hline $\begin{array}{l}\text { History of percutaneous coronary } \\
\text { intervention }\end{array}$ & 0.33 & $0.04-2.40$ & 0.2712 \\
\hline History of congestive heart failure & 1.01 & $0.30-3.34$ & 0.9909 \\
\hline History of atrial fibrillation & 1.12 & $0.39-3.22$ & 0.8284 \\
\hline History of AICD/pacemaker & 0.00 & $0.00-\operatorname{lnf}$ & 0.9853 \\
\hline $\begin{array}{l}\text { History of hypertension requiring } \\
\text { treatment }\end{array}$ & 0.92 & $0.50-1.70$ & 0.7924 \\
\hline History of heart valve disease & 0.93 & $0.28-3.07$ & 0.9020 \\
\hline History of COPD & 0.87 & $0.33-2.24$ & 0.7656 \\
\hline History of asthma & 0.00 & $0.00-\operatorname{lnf}$ & 0.9826 \\
\hline History of respiratory failure & 0.00 & $0.00-\operatorname{Inf}$ & 0.9847 \\
\hline $\begin{array}{l}\text { History of restrictive pulmonary } \\
\text { disease }\end{array}$ & 3.58 & $0.42-30.41$ & 0.2424 \\
\hline History of cirrhosis & 1.42 & $0.18-10.99$ & 0.7378 \\
\hline History of peptic ulcer disease & 1.01 & $0.13-7.66$ & 0.9949 \\
\hline History of renal insufficiency & 1.68 & $0.69-4.09$ & 0.2551 \\
\hline History of dialysis & 3.07 & $0.37-25.48$ & 0.2997 \\
\hline $\begin{array}{l}\text { History of non-cancerous } \\
\text { hematology disease }\end{array}$ & 0.00 & $0.00-\operatorname{Inf}$ & 0.9847 \\
\hline History of anemia & 0.00 & $0.00-\operatorname{lnf}$ & 0.9881 \\
\hline History of ITP & 0.00 & $0.00-\operatorname{Inf}$ & 0.9896 \\
\hline $\begin{array}{l}\text { History of peripheral vascular } \\
\text { disease }\end{array}$ & 0.73 & $0.22-2.40$ & 0.6011 \\
\hline History of VTE & 0.53 & $0.07-3.96$ & $0.537 \mid$ \\
\hline History of DVT & 0.00 & $0.00-\operatorname{lnf}$ & 0.9850 \\
\hline History of PE & 1.25 & $0.16-9.60$ & 0.8308 \\
\hline History of diabetes & 0.58 & $0.21-1.66$ & 0.3122 \\
\hline History of hyperthyroidism & 4.30 & $0.49-37.63$ & 0.1873 \\
\hline History of hypothyroidism & 0.30 & $0.04-2.21$ & 0.2368 \\
\hline History of stroke & 1.19 & $0.36-3.96$ & 0.7802 \\
\hline History of TIA & 1.34 & $0.31-5.76$ & 0.6975 \\
\hline History of dementia & 0.00 & $0.00-\operatorname{lnf}$ & 0.9881 \\
\hline History of neuromuscular disease & 0.00 & $0.00-\operatorname{lnf}$ & 0.9896 \\
\hline History of seizures & 1.18 & $0.15-9.03$ & 0.8745 \\
\hline AIDS & 0.00 & $0.00-\operatorname{lnf}$ & 0.9863 \\
\hline $\begin{array}{l}\text { Immunosuppression within past } 6 \\
\text { months }\end{array}$ & 7.19 & $0.73-70.52$ & 0.0905 \\
\hline History of rheumatic disease & 1.78 & $0.23-14.00$ & $0.584 I$ \\
\hline History of rheumatoid arthritis & 1.78 & $0.23-14.00$ & $0.584 I$ \\
\hline History of SLE & 0.00 & $0.00-\operatorname{Inf}$ & 0.9896 \\
\hline History of heart transplant & 0.00 & $0.00-\operatorname{lnf}$ & 0.9853 \\
\hline History of liver transplant & $\operatorname{lnf}$ & $0.00-\operatorname{lnf}$ & $0.984 I$ \\
\hline History of renal transplant & 7.19 & $0.73-70.52$ & 0.0905 \\
\hline History of cancer & 0.16 & $0.02-1.21$ & 0.0761 \\
\hline History of metastases cancer & 0.00 & $0.00-\operatorname{lnf}$ & 0.9863 \\
\hline History of head neck cancer & 0.00 & $0.00-\operatorname{Inf}$ & 0.9890 \\
\hline
\end{tabular}

(Continued)
Table 5 (Continued).

\begin{tabular}{|l|l|l|l|}
\hline Variables & OR* & $\mathbf{9 5 \%} \mathbf{~ C I}$ & P value \\
\hline History of esophagus cancer & 0.00 & $0.00-\operatorname{Inf}$ & 0.9863 \\
History of lung cancer & 0.00 & $0.00-\operatorname{Inf}$ & 0.9820 \\
History of breast cancer & 0.00 & $0.00-\operatorname{Inf}$ & 0.9844 \\
History of liver cancer & 0.00 & $0.00-\operatorname{Inf}$ & 0.9853 \\
History of stomach cancer & 0.00 & $0.00-\operatorname{Inf}$ & $0.988 \mathrm{I}$ \\
History of bladder cancer & 0.00 & $0.00-\operatorname{Inf}$ & 0.9858 \\
History of kidney cancer & 0.00 & $0.00-\operatorname{Inf}$ & 0.9858 \\
History of colon cancer & 0.00 & $0.00-\operatorname{Inf}$ & 0.9847 \\
History of melanoma & 0.00 & $0.00-\operatorname{Inf}$ & 0.9873 \\
History of ovary cancer & 0.00 & $0.00-\operatorname{Inf}$ & 0.9853 \\
History of uterus cancer & 0.00 & $0.00-\operatorname{Inf}$ & 0.9896 \\
History of prostate cancer & 1.01 & $0.13-7.66$ & 0.9949 \\
\hline History of chemotherapy & 0.00 & $0.00-\operatorname{Inf}$ & $0.988 \mathrm{I}$ \\
Radiation therapy within past 6 & 0.00 & $0.00-\operatorname{Inf}$ & 0.9853 \\
months & \multicolumn{3}{|l}{} \\
\hline
\end{tabular}

Note: *Compared with patients without the specific comorbidity.

Abbreviations: ICU, intensive care unit; OR, odds ratio; $\mathrm{Cl}$, confidence interval; AICD, automatic implantable cardioverter defibrillator; Inf, infinity; COPD, chronic obstructive pulmonary disease; ITP, immune thrombocytopenic purpura; VTE, venous thromboembolism; DVT, deep vein thrombosis; PE, pulmonary embolism; TIA, transient ischemic attack; AIDS, acquired immune deficiency syndrome; SLE, systemic lupus erythematosus.

higher risk of mortality. In our study, we also found that compared with patients without ruptured AA, the risk of ICU mortality of patients with ruptured AA increased (OR $3.31,95 \%$ CI 1.53-7.18). Another reason could be the different surgery treatments received, since EVAR was found to have lower perioperative mortality than open surgery. ${ }^{30}$ Although in our study detailed information about surgical treatment was unavailable, given the study period was between 2014 and 2015, and most cases received elective surgery, they might be less likely to receive open surgery. We also found that patients with longer length of ICU stay had worse prognosis, which was consistent with the study conducted by Gavali et al. ${ }^{31}$

Among the many variables, only a few factors that related to the condition itself were significantly associated with prognosis, including elective surgery, with aortic dissection, rupture of $\mathrm{AA}$, and type of $\mathrm{AA}$, while none of the comorbidities show a significant association. These results suggested at least the short-term prognosis was mainly determined by the condition itself. APACHE IVa was also found to be associated with ICU mortality of the study population, and ROC curve analysis showed it had good discriminatory capability. This is consistent with Kabbani et $\mathrm{al}^{32}$ study where they found APACHE III 
Table 6 Predictive Performance of APACHE IVa for ICU Mortality

\begin{tabular}{|l|l|l|l|l|l|}
\hline & AUC & $\mathbf{9 5 \%} \mathbf{~ C l}$ & Best Threshold & Specificity & Sensitivity \\
\hline All patient & 0.9176 & $0.8789-0.9390$ & 58.5 & 0.7399 & 0.9767 \\
\hline $\begin{array}{l}\text { Rupture of aortic aneurysm } \\
\text { No }\end{array}$ & 0.9158 & $0.8704-0.9419$ & 58.5 & 0.7653 & 0.8209 \\
Yes & 0.8795 & $0.6879-0.9614$ & 94 & 0.9706 \\
\hline $\begin{array}{l}\text { Type of aortic aneurysm } \\
\text { Abdominal aortic aneurysm } \\
\text { Thoracic aortic aneurysm }\end{array}$ & 0.9233 & $0.8440-0.9649$ & 65.5 & 0.8438 & 1.0000 \\
\hline
\end{tabular}

Abbreviations: APACHE, Acute Physiology And Chronic Health Evaluation; ICU, intensive care unit; AUC, area under the receiver operating characteristic curve; Cl, confidence interval.

score on ICU admission was an excellent discriminator of hospital mortality (AUC 0.92, 95\% CI 0.83-1.00) for patients after open thoracoabdominal and open AAA repair. As far as know, there is no study evaluating the predictive performance of APACHE IVa in AAA and TAA patients after surgical treatment, and thus our finding could be seen as a validation of this scoring system in AA patients.

The strengths of the study included a multiple centers design and the updated data. The relatively large sample size also increased the power of the study. In addition, benefiting from the great abundance of variables in the database, various variables about baseline characteristics and comorbidities were investigated in the study. However, the study inevitably had some limitations. First, as a retrospective study, all the data we used were directly extracted from the available database without validation, and therefore measurement error should be considered as the main source of bias in the study. For example, it is possible that a diagnosis of AAA, TAA, or a TAA that also involves the aortic valve could be entered into the database incorrectly, since it was usually entered by nurses instead of clinicians. Second, since the database only contained data during the ICU hospitalization, information about surgical treatment before ICU was unavailable. We, therefore, applied strict inclusion criteria on the study population, and only included patients who were with a primary diagnosis of AAA or TAA and were directly admitted to ICU from operating room, recovery room, or PACU. Another concern was the potential selection bias of the study population. Since not every ICU or hospital participated in the eICU program, AA patients admitted to those unanticipated ICU/hospitals would always be missed in our study. Nevertheless, further studies are needed to validate the findings in our study.

\section{Conclusion}

Prognosis of aortic aneurysm patients admitted to ICU postoperatively is yet to improve, and factors associated with prognosis are mainly related to the condition itself, while APACHE IVa is a reliable tool for prognosis prediction.

\section{Funding}

This work was supported by the National Natural Science Funds for Young Scholars of China [grant number 81800416] and the General Program of National Natural Science Foundation of China [grant number 82070495].

\section{Disclosure}

The authors declare that there are no conflicts of interest.

\section{References}

1. Creager MA, Belkin M, Bluth EI, et al. 2012 ACCF/AHA/ACR/SCAI/ SIR/STS/SVM/SVN/SVS key data elements and definitions for peripheral atherosclerotic vascular disease: a report of the American College of Cardiology Foundation/American Heart Association Task Force on clinical data standards (writing committee to develop clinical data standards for peripheral atherosclerotic vascular disease). $J \mathrm{Am}$ Coll Cardiol. 2012;59(3):294-357. doi:10.1016/j.jacc.2011.10.860

2. Hiratzka LF, Bakris GL, Beckman JA, et al. 2010 ACCF/AHA/AATS/ ACR/ASA/SCA/SCAI/SIR/STS/SVM guidelines for the diagnosis and management of patients with thoracic aortic disease: a report of the American College of Cardiology Foundation/American Heart Association Task Force on Practice Guidelines, American Association for Thoracic Surgery, American College of Radiology, American Stroke Association, Society of cardiovascular anesthesiologists, society for cardiovascular angiography and interventions, society of interventional radiology, society of thoracic surgeons, and society for vascular medicine. Circulation. 2010;121(13):e266-e369. doi:10.1161/CIR.0b013e3181d4739e

3. Johnston KW, Rutherford RB, Tilson MD, Shah DM, Hollier L, Stanley JC. Suggested standards for reporting on arterial aneurysms. Subcommittee on reporting standards for arterial aneurysms, Ad Hoc committee on reporting standards, society for vascular surgery and North American chapter, international society for cardiovascular surgery. J Vasc Surg. 1991;13 (3):452-458. doi:10.1067/mva.1991.26737

4. Kent KC. Clinical practice. Abdominal aortic aneurysms. $N$ Engl $J$ Med. 2014;371(22):2101-2108. doi:10.1056/NEJMcp1401430 
5. Ashton HA, Buxton MJ, Day NE, et al. The Multicentre Aneurysm Screening Study (MASS) into the effect of abdominal aortic aneurysm screening on mortality in men: a randomised controlled trial. Lancet. 2002;360(9345):1531-1539. doi:10.1016/S0140-6736(02)11522-4

6. Norman PE, Jamrozik K, Lawrence-Brown MM, et al. Population based randomised controlled trial on impact of screening on mortality from abdominal aortic aneurysm. BMJ. 2004;329(7477):1259. doi:10.1136/bmj.38272.478438.55

7. Lindholt JS, Juul S, Fasting H, Henneberg EW. Screening for abdominal aortic aneurysms: single centre randomised controlled trial. BMJ. 2005;330(7494):750. doi:10.1136/bmj.38369.620162.82

8. Ashton HA, Gao L, Kim LG, Druce PS, Thompson SG, Scott RA. Fifteen-year follow-up of a randomized clinical trial of ultrasonographic screening for abdominal aortic aneurysms. Br J Surg. 2007;94(6):696-701. doi:10.1002/bjs.5780

9. Itani Y, Watanabe S, Masuda Y, et al. Measurement of aortic diameters and detection of asymptomatic aortic aneurysms in a mass screening program using a mobile helical computed tomography unit. Heart Vessels. 2002;16(2):42-45. doi:10.1007/s380-002-8315-1

10. Kalsch H, Lehmann N, Mohlenkamp S, et al. Body-surface adjusted aortic reference diameters for improved identification of patients with thoracic aortic aneurysms: results from the population-based Heinz Nixdorf Recall study. Int J Cardiol. 2013;163(1):72-78. doi:10.1016/ j.ijcard.2011.05.039

11. Swerdlow NJ, Wu WW, Schermerhorn ML. Open and endovascular management of aortic aneurysms. Circ Res. 2019;124(4):647-661. doi:10.1161/CIRCRESAHA.118.313186

12. Brady AR, Fowkes FG, Greenhalgh RM, Powell JT, Ruckley CV, Thompson SG. Risk factors for postoperative death following elective surgical repair of abdominal aortic aneurysm: results from the UK small aneurysm trial. On behalf of the UK small aneurysm trial participants. Br J Surg. 2000;87(6):742-749. doi:10.1046/j.1365-2168.2000.01410.x

13. Dillavou ED, Muluk SC, Makaroun MS. Improving aneurysm-related outcomes: nationwide benefits of endovascular repair. J Vasc Surg. 2006;43(3):446-51; discussion 451-2. doi:10.1016/j.jvs.2005.11.017

14. Lee WA, Carter JW, Upchurch G, Seeger JM, Huber TS. Perioperative outcomes after open and endovascular repair of intact abdominal aortic aneurysms in the United States during 2001. J Vasc Surg. 2004;39(3):491-496. doi:10.1016/j.jvs.2003.12.001

15. Lieberg J, Pruks LL, Kals M, Paapstel K, Aavik A, Kals J. Mortality after elective and ruptured abdominal aortic aneurysm surgical repair: 12-year single-center experience of estonia. Scand J Surg. 2018;107 (2):152-157. doi:10.1177/1457496917738923

16. Kuckelman J, Niven A, Martin MJ. Postoperative intensive care unit management after ruptured abdominal aortic aneurysm. In: Starnes BW, Mehta M, Veith FJ, editors. Ruptured Abdominal Aortic Aneurysm: The Definitive Manual. Springer International Publishing; 2017:273-310.

17. Lawlor DK, Lovell MB, DeRose G, Forbes TL, Harris KA. Is intensive care necessary after elective abdominal aortic aneurysm repair? Can J Surg. 2004;47(5):359-363.

18. Bisgaard J, Jørgensen HK, Gilsaa T, Ronholm E, Toft P. Aortic aneurysm disease versus aortic occlusive disease: differences in postoperative ICU requirements after open elective abdominal aortic surgery. Crit Care. 2012;16(1):P470. doi:10.1186/cc11077
19. Hicks CW, Alshaikh HN, Zarkowsky D, Bostock IC, Nejim B, Malas MB. Intensive care unit admission after endovascular aortic aneurysm repair is primarily determined by hospital factors, adds significant cost, and is often unnecessary. J Vasc Surg. 2018;67 (4):1091-1101.e4. doi:10.1016/j.jvs.2017.07.139

20. Pollard TJ, Johnson AEW, Raffa JD, Celi LA, Mark RG, Badawi O. The eICU collaborative research database, a freely available multi-center database for critical care research. Sci Data. 2018. doi:10.1038/sdata.2018.178

21. Nakano M, Kimura N, Matsumoto H, et al. Characteristics of abdominal aortic aneurysm in Japanese patients aged 50 years or younger. Ann Vasc Dis. 2017;10(2):119-124. doi:10.3400/avd.oa.16-00083

22. Forouzanfar MM, Barazesh F, Hashemi B, Safari S. Characteristics and outcome of abdominal aortic aneurysm in emergency department; a 10-year Cross-sectional Study. Arch Acad Emerg Med. 2020;8(1):e4.

23. Joyce JW, Fairbairn JF 2nd, Kincaid OW, Juergen JL. Aneurysms of the thoracic aorta. A clinical study with special reference to prognosis. Circulation. 1964;29:176-181. doi:10.1161/01.CIR.29.2.176

24. Gao Z, Xiong J, Chen Z, et al. Gender differences of morphological and hemodynamic characteristics of abdominal aortic aneurysm. Biol Sex Differ. 2020;11(1):41. doi:10.1186/s13293-020-00318-3

25. Salem MK, Rayt HS, Hussey G, et al. Should Asian men be included in abdominal aortic aneurysm screening programmes? Eur J Vasc Endovasc Surg. 2009;38(6):748-749. doi:10.1016/j.ejvs.2009.07.012

26. Lederle FA, Johnson GR, Wilson SE, et al. The aneurysm detection and management study screening program: validation cohort and final results. Aneurysm detection and management veterans affairs Cooperative Study Investigators. Arch Intern Med. 2000;160 (10):1425-1430. doi:10.1001/archinte.160.10.1425

27. Kent KC, Zwolak RM, Egorova NN, et al. Analysis of risk factors for abdominal aortic aneurysm in a cohort of more than 3 million individuals. J Vasc Surg. 2010;52(3):539-548. doi:10.1016/j.jvs.20 10.05 .090

28. Jonker FH, Verhagen HJ, Lin PH, et al. Outcomes of endovascular repair of ruptured descending thoracic aortic aneurysms. Circulation. 2010;121(25):2718-2723. doi:10.1161/CIRCULATIONAHA.109.90 8871

29. Laukontaus SJ, Lepantalo M, Hynninen M, Kantonen I, Pettila V. Prediction of survival after 48-h of intensive care following open surgical repair of ruptured abdominal aortic aneurysm. Eur J Vasc Endovasc Surg. 2005;30(5):509-515. doi:10.1016/j.ejvs.2005.06.013

30. Giles KA, Pomposelli F, Hamdan A, Wyers M, Jhaveri A, Schermerhorn ML. Decrease in total aneurysm-related deaths in the era of endovascular aneurysm repair. J Vasc Surg. 2009;49(3):543-50; discussion 550-1. doi:10.1016/j.jvs.2008.09.067

31. Gavali H, Mani K, Tegler G, Kawati R, Covaciu L, Wanhainen A. Editor's choice - prolonged ICU length of stay after AAA repair: analysis of time trends and long-term outcome. Eur J Vasc Endovasc Surg. 2017;54(2):157-163. doi:10.1016/j.ejvs.2017.05.014

32. Kabbani LS, Escobar GA, Knipp B, et al. APACHE III score on ICU admission predicts hospital mortality after open thoracoabdominal and open abdominal aortic aneurysm repair. Ann Vasc Surg. 2010;24(8):1060-1067. doi:10.1016/j.avsg.2010.07.011

International Journal of General Medicine

\section{Publish your work in this journal}

The International Journal of General Medicine is an international, peer-reviewed open-access journal that focuses on general and internal medicine, pathogenesis, epidemiology, diagnosis, monitoring and treatment protocols. The journal is characterized by the rapid reporting of reviews, original research and clinical studies

across all disease areas. The manuscript management system is completely online and includes a very quick and fair peer-review system, which is all easy to use. Visit http://www.dovepress.com/ testimonials.php to read real quotes from published authors. 